\title{
Disyunción del anillo mitral como marcador de riesgo en prolapso de válvula mitral
}

\author{
The mitral annulus disjunction as a risk marker in mitral valve prolapse
}

\author{
Keerby Hernández ${ }^{1 *}$, Juan F. Agudelo-Uribe², Juan D. Ramírez-Barrea², Pedro Abad-Díaz³, \\ Rafael Correa-Velásquez ${ }^{2}$ y Gloria Sáenz-Jaramillo \\ ${ }^{1}$ Departamento de Cardiología Pediátrica, Universidad Pontificia Bolivariana, Clínica CardioVID; ${ }^{2}$ Servicio de Electrofisiología, Clínica CardioVID; \\ ${ }^{3}$ Servicio de Imagen Cardiovascular, SURA. Medellín, Colombia
}

\begin{abstract}
Resumen
La disyunción del anillo valvular mitral es una anormalidad estructural que consiste en un desplazamiento auricular del punto de articulación de la válvula mitral, lo que lleva a una relación espacial alterada entre la válvula y la pared ventricular posterior adyacente. Los estudios han demostrado una relación entre la disyunción del anillo mitral, las arritmias ventriculares y la degeneración fibrosa miocárdica, lo que incrementa el riesgo de muerte súbita, en especial en mujeres jóvenes. Se presenta el caso de una mujer de 30 años, sin antecedentes relevantes, con palpitaciones frecuentes, en quien se documentó un aumento del automatismo ventricular con incremento progresivo de la carga arrítmica hasta el 20\% a pesar del manejo farmacológico. En la resonancia cardiaca se apreció un desplazamiento de $15 \mathrm{~mm}$ hacia la aurícula de las valvas de la mitral compatible con disyunción del anillo mitral, además de focos de realce tardío de distribución mesocárdica, no coronariana. Ante los hallazgos y la persistencia de los síntomas, se llevó a estudio electrofisiológico, mapeo 3D y ablación. En la ecografía intracardiaca se apreciaron dos regiones hiperecoicas, en la longitud del músculo papilar anterior y en el mesocardio de la base del músculo papilar posterior; ambos focos relacionados con los sitios morfológicos de interés, en los cuales se aplicó energía de radiofrecuencia. Durante el procedimiento presentó un episodio de fibrilación ventricular que se consideró una extrasistolia ventricular maligna, por lo que se implantó un cardiodesfibrilador para prevención de muerte súbita. Se revisa la literatura y se analizan las relaciones fisiopatológicas existentes entre la disyunción del anillo mitral, los complejos ventriculares prematuros y el riesgo de muerte súbita.
\end{abstract}

Palabras clave: Ablación por catéter. Fibrilación ventricular. Muerte súbita. Taquicardia ventricular. Válvula mitral.

\begin{abstract}
Disjunction of the mitral valve annulus is a structural abnormality consisting of an atrial displacement of the articulation point of the mitral valve, leading to an altered spatial relationship between the valve and the adjacent posterior ventricular wall. Studies have shown the relationship between mitral annular disjunction, ventricular arrhythmias, and myocardial fibrous degeneration, which increases the risk of sudden death, especially among young women. The case of a 30-year-old woman
\end{abstract}

\footnotetext{
Correspondencia:

*Keerby Hernández

E-mail: Keerby.cardioped@gmail.com

Fecha de recepción: 29-07-2020

Fecha de aceptación: 04-09-2020

DOI: 10.24875/ACM.200003661

Disponible en internet: 23-11-2020

Arch Cardiol Mex. 2021;91(3):347-354 www.archivoscardiologia.com

1405-9940 / (C) 2020 Instituto Nacional de Cardiología Ignacio Chávez. Publicado por Permanyer. Este es un artículo open access bajo la licencia CC BY-NC-ND (http://creativecommons.org/licenses/by-nc-nd/4.0/).
} 
with no relevant pathological history with frequent palpitations is presented, in whom an increase in ventricular automatism with a progressive increase in the arrhythmic load of up to $20 \%$ with a weight of pharmacological management is documented. In a cardiac magnetic resonance, a $15 \mathrm{~mm}$ displacement towards the atrium of the mitral leaflets compatible with mitral annular disjunction and late enhancement foci of mesocardial, non-coronary distribution were observed. Given the findings and the persistence of symptoms, an electrophysiological study, 3D mapping, and ablation were performed. Two hyperechoic regions were seen on intracardiac ultrasound, one in the length of the anterior papillary muscle and the other in the mesocardium of the base of the posterior papillary muscle. Both foci related to the morphological sites of interest in which radiofrequency energy was applied. During the procedure presented an episode of ventricular fibrillation. A malignant ventricular extrasystole was considered and therefore a cardio defibrillator was implanted for the secondary prevention of sudden death. The literature was reviewed and the specific pathophysiological relationships between mitral annular disjunction, premature ventricular complexes, and risk of sudden death were analyzed. The role of electrophysiological study and ablation in symptomatic patients refractory to pharmacological treatment is described.

Key words: Catheter ablation. Ventricular fibrillation. Sudden death. Ventricular tachycardia. Mitral valve.

\section{Introducción}

La disyunción del anillo mitral (DAM) fue descrita hace tres décadas por Bharati, et al. ${ }^{1}$, quienes documentaron el caso de un médico de 45 años con antecedentes de palpitaciones, clic medio diastólico, complejos ventriculares prematuros frecuentes y episodios de taquicardia ventricular no sostenida, quien murió súbitamente. Los hallazgos patológicos post mortem revelaron una válvula mitral mixomatosa, redundante, con inserción atrial de la valva posterior.

En estudios de autopsia de 900 corazones se encontró una prevalencia del $3 \%$ de la degeneración valvular mitral, y entre ellos, el 92\% presentaron DAM. Típicamente eran pacientes jóvenes, por lo que se sugirió que el desarrollo de degeneración del tejido conectivo era secundario a la disfunción del anillo fibroso mitral². Eriksson, et al. $^{3}$ evaluaron pacientes sometidos a reparación de la válvula mitral por degeneración mixomatosa, utilizando un protocolo ecocardiográfico transesofágico, y observaron una disyunción anular (valor medio de $10 \mathrm{~mm}$ ) en la base de la valva posterior en el $98 \%$ de los pacientes con enfermedad avanzada; demostraron que su reconocimiento por esta modalidad de imagen modificaba la técnica de reparación para optimizar los resultados a largo plazo.

Desde entonces, ha habido un creciente interés en la investigación de las relaciones existentes entre las anormalidades anatómicas del aparato valvular mitral y su potencial arritmogénico.

El movimiento anormal de la región posterior del anillo mitral secundario a la inserción anómala de las valvas favorece un movimiento curvilíneo durante la sístole ventricular, sin ningún componente anterior e hipermovilidad del aparato subvalvular, lo que resulta en una apariencia «rizada» cuando se evalúa en imágenes ecocardiográficas bidimensionales ${ }^{4}$. La pérdida de la sincronía en el movimiento se ha relacionado con el desarrollo de fibrosis en el ventrículo izquierdo, por excesiva movilidad y tracción continua. Este fenómeno incrementa el estrés de la célula miocárdica adyacente a la válvula, afectando principalmente las cuerdas tendinosas y los músculos papilares. Tal compromiso fibroso ha sido demostrado en estudios histopatológicos y por caracterización tisular con resonancia magnética, principalmente con las secuencias de realce tardío tras la administración de gadolinio.

La fibrosis generada por el estrés continuado no solo altera la geometría ventricular, sino que se constituye en un sustrato para la inestabilidad eléctrica y es desencadenante de arritmias potencialmente letales. La combinación del sustrato (fibrosis) y el estrés mecánico continuo promueve la génesis de complejos ventriculares prematuros de los músculos papilares o de los fascículos, que pueden desencadenar fibrilación ventricular, fundamentalmente por estar asociados a compromiso de las fibras de Purkinje ${ }^{5-7}$. La ablación con catéter de los focos arritmogénicos desempeña un papel importante para reducir la carga arrítmica y disminuir las descargas apropiadas e inapropiadas de un desfibrilador ${ }^{8}$.

\section{Presentación del caso}

Se trata de una mujer de 30 años, sin antecedentes patológicos ni familiares de importancia. Tiene un cuadro de varios años de evolución consistente en palpitaciones que se presentan de manera súbita, sin relación con el esfuerzo físico y que ceden de manera espontánea, sin evidencia de síncope. Consulta en un centro de cuarto nivel de complejidad, especializado en el manejo de enfermedades cardiovasculares. En la exploración física, sus constantes vitales se encuentran dentro 
de los parámetros normales, con un soplo holosistólico en focos de la base como único hallazgo patológico.

La evaluación inicial incluyó un electrocardiograma de 12 derivaciones y un monitoreo Holter de 24 horas en el que se observaron latidos ventriculares prematuros de varias morfologías dispuestos en forma aislada, con periodos de bigeminismo, trigeminismo, dupletas y taquicardia ventricular monomórfica y pleomórfica de hasta seis latidos, con una carga arrítmica inicial del $6 \%$ y con conducción auriculoventricular e intraventricular normal. El ecocardiograma transtorácico mostró una válvula mitral mixomatosa, con valvas móviles y redundantes, de $6 \mathrm{~mm}$ de grosor, con prolapso de los segmentos A2 y P2, desplazamiento más allá del plano valvular de $0,6 \mathrm{~cm}$ del segmento $A 2$ y $0,5 \mathrm{~cm}$ del segmento P2 que genera una insuficiencia moderada. El Doppler color mostró un flujo de regurgitación hacia la pared posterior y hasta el techo de la aurícula izquierda, con un ancho de vena contracta de $0,5 \mathrm{~cm}$. La aurícula y el ventrículo izquierdos estaban muy dilatados, con un diámetro de fin de diástole del ventrículo izquierdo de $3,8 \mathrm{~cm} / \mathrm{m}^{2}$, contractilidad miocárdica global y segmentaria normal, y fracción de eyección del $60 \%$ evaluada por Simpson.

Se inició manejo farmacológico con un betabloqueador, pero los síntomas persistieron y se apreció un incremento de la carga arrítmica hasta el $20 \%$. Se decidió realizar un estudio electrofisiológico con mapeo 3D (NavX® Ensite ${ }^{\circledR}$, St. Jude Medical, St. Paul, MN, USA) y ablación. Durante el procedimiento se documentaron tres morfologías de extrasístoles cuyo origen se localizó hacia la continuidad mitroaórtica y paraseptal. Se practicó modulación con energía de radiofrecuencia del sustrato arrítmico.

Durante el seguimiento ambulatorio la paciente persistió sintomática, aunque sin documentarse síncope. Se inició propafenona a dosis adecuadas, con buena adherencia farmacológica. Se ampliaron los estudios con una resonancia magnética cardiaca que mostró una discordancia entre la inserción del anillo valvular mitral (Fig. $1 \mathrm{~A}$ y B) y un desplazamiento auricular de los puntos de inserción de la valva de $15 \mathrm{~mm}$, compatible con DAM. Se apreciaron además focos de realce tardío de distribución mesocárdica, no coronariana (Fig. 2 C). Se realizó un nuevo Holter de 12 derivaciones donde se observa la morfología de las extrasístoles (Fig. 2 A).

Ante la persistencia de los síntomas, la refractariedad al manejo médico, la alta carga arrítmica y el potencial maligno, se decidió llevar a nuevo estudio electrofisiológico con mapeo 3D y ablación con energía de radiofrecuencia.

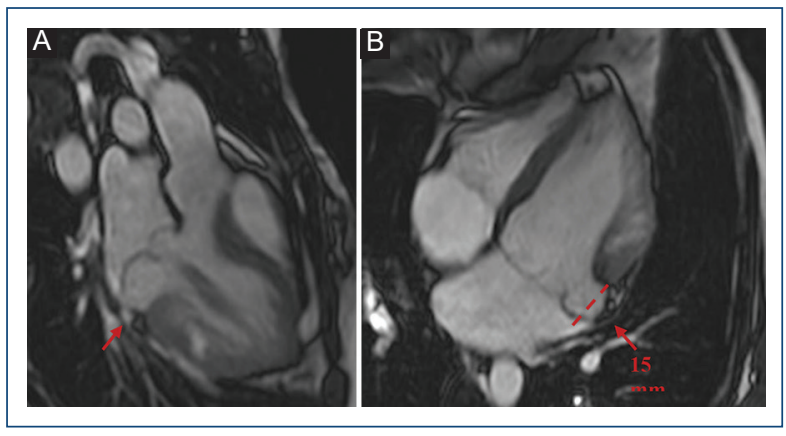

Figura 1. Imágenes de resonancia magnética, secuencias cine de tres cámaras (A) y cuatro cámaras (B). Se observa en una adquisición a nivel del tracto de salida del ventrículo izquierdo una aurícula izquierda dilatada, con un área de $16 \mathrm{~cm} / \mathrm{m}^{2}$. El ventrículo izquierdo está dilatado sin hipertrofia, con discordancia entre el anillo mitral y las valvas de la válvula mitral, secundario a un desplazamiento anormal de los puntos de inserción hacia la aurícula izquierda. Distancia de $15 \mathrm{~mm}$.

Bajo sedación superficial con infusión de remifentanilo suministrada por el anestesiólogo cardiovascular, se inició el estudio en ritmo sinusal con extrasístoles ventriculares de diferente morfología, predominando (M1) positivas en DII, DIII y aVF, con bloqueo de rama derecha y negativas en DI y aVL (Fig. 2 B). Los electrocatéteres diagnósticos (Inquiry®, St. Jude Medical, St Paul, MN, USA) cuadripolar y decapolar fueron posicionados en el ápex del ventrículo derecho y en el seno coronario, respectivamente. A través de una punción en la arteria femoral común se avanzó el catéter de ablación bidireccional con sensor de contacto (Tacticath ${ }^{\circledR}$ Quartz Contact Force, St. Jude Medical, St Paul, MN, USA), que se posicionó en el ventrículo izquierdo.

Bajo visión fluoroscópica y ecográfica se avanzó una camisa deflectable (Agilis ${ }^{\circledR}$, St. Jude Medical, St Paul, MN, USA), y con una aguja de Brockenbrough de $98 \mathrm{~cm}$ (BRK®, St. Jude Medical, St Paul, MN, USA) se realizó una punción transeptal sin complicaciones. A través de esta se pasó un catéter de mapeo de alta densidad (Advisor ${ }^{\circledR}$ HD Grid mapping Catheter, St. Jude Medical, St Paul, MN, USA), que se avanzó también hasta el ventrículo izquierdo, y con un sistema 3D (Ensite ${ }^{\circledR}$ Presicion Cardiac Mapping System, St. Jude Medical, St Paul, MN, USA) se trazó un mapa anatómico tridimensional de activación del ventrículo izquierdo con énfasis en la pared lateral, porción basal, correspondiente a la primera morfología (M1). Simultáneamente se mapeó una segunda morfología (M2) con eje hacia superior y positiva en DI y aVL. 


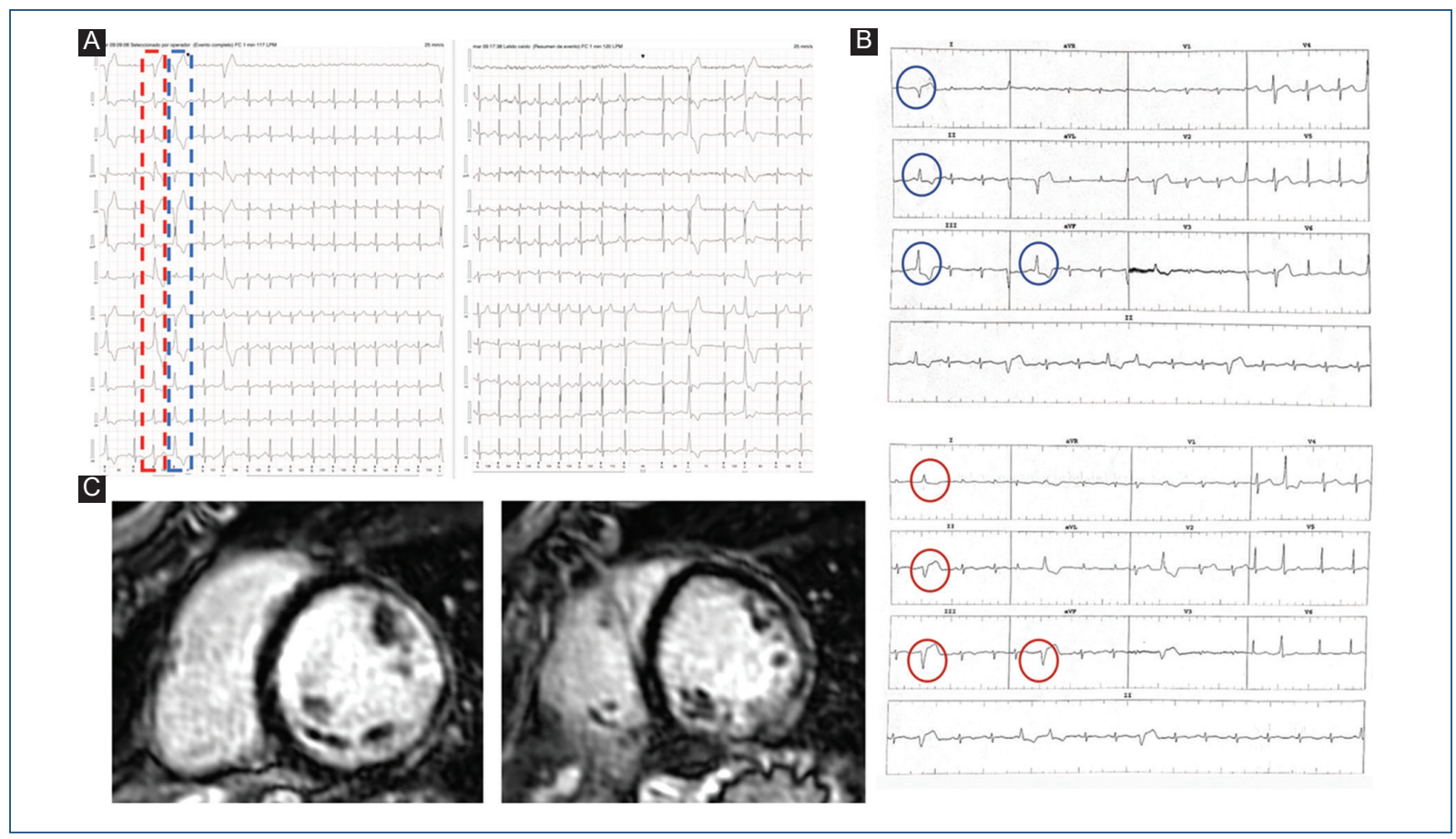

Figura 2. A: registro Holter de 12 derivaciones en el que se observan extrasístoles ventriculares con tres morfologías predominantes. B: electrocardiograma de superficie durante el estudio electrofisiológico en el que se observa una morfología claramente predominante. M1: morfología de bloqueo de rama derecha, positivas en II, III, aVF y negativas en I y aVL. M2: con eje hacia superior y positiva en DI y aVL. Carga arrítmica del 15\%. C: imagen de resonancia magnética cardiaca, secuencias de realce tardío tras la administración de gadolinio con focos fibróticos de distribución no coronariana y de localización en los segmentos 7 y 8.

De la morfología mas frecuente (M1) se logró obtener precocidad de hasta $-69 \mathrm{~ms}$ (con respecto al inicio del QRS), con potenciales fraccionados en la región lateral y basal, donde además el mapa de voltaje mostró zonas de transición. También se apreciaron potenciales de Purkinje y potenciales muy fraccionados en los sitios de mayor precocidad.

Paralelamente, en el ecocardiograma intracardiaco (Viewflex ${ }^{\circledR}$ intracardiac Echo Probe, St. Jude Medical, St Paul, MN, USA) se apreciaron dos áreas hiperecoicas, refringentes, sugestivas de cicatriz homogénea, una en toda la longitud del músculo papilar anterior y la otra en la región mesocárdica en la base del músculo papilar posterior. Se decidió aplicar energía de radiofrecuencia con el fin de homogenizar la cicatriz del músculo anterior, iniciando en el área de mayor precocidad y rodeándola por completo para consolidar. Durante una de las aplicaciones se desarrolló una taquicardia ventricular monomórfica que degeneró en fibrilación ventricular (Fig. 3) y requirió desfibrilación, retornando a ritmo sinusal. Una vez en condiciones basales, se completó el protocolo de estimulación desde el ventrículo hasta tres extraestímulos y dos longitudes de ciclo, sin lograr inducir arritmia alguna. Se utilizó además infusión de dobutamina a razón de $15 \mu \mathrm{g} / \mathrm{kg} / \mathrm{min}$ y bajo efecto de esta se repitió el protocolo de estimulación ventricular. La no inducibilidad de la arritmia clínica y la desaparición de las extrasístoles ventriculares fueron los puntos finales para la ablación.

Se consideró que se trataba de una paciente con alto riesgo de arritmias letales, con inducción de fibrilación ventricular durante el procedimiento, con patología estructural cardiaca que involucra el complejo valvular mitral y especialmente los músculos papilares (muy posiblemente la red de Purkinje al inducir tan fácilmente fibrilación ventricular), y con fibrosis importante, que constituyen un sustrato arritmogénico, por lo cual se decidió implantar un cardiodesfibrilador para prevención primaria de muerte súbita.

\section{Discusión}

La válvula mitral deriva de los cojines endocárdicos. Su funcionamiento adecuado requiere la perfecta 


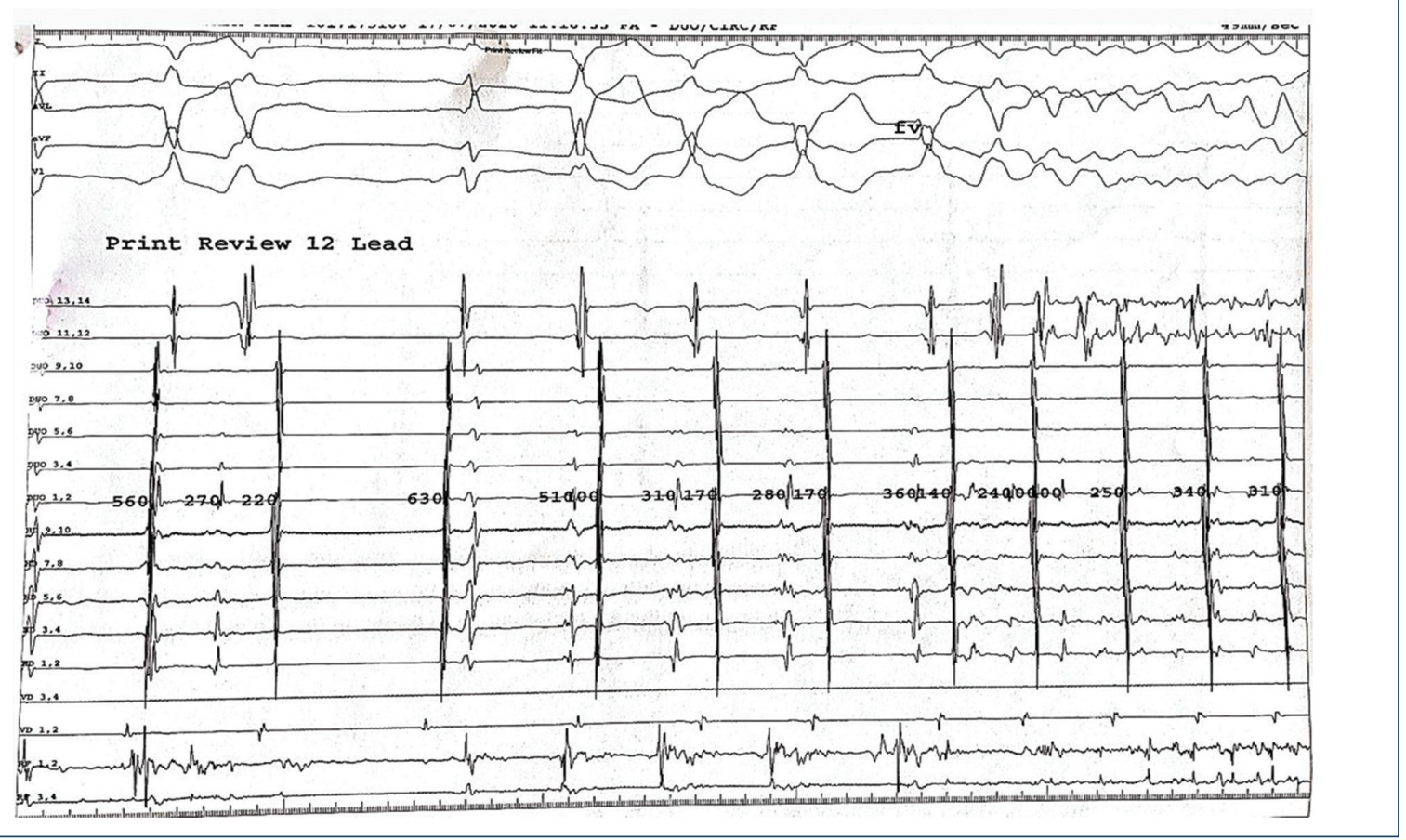

Figura 3. Registro de polígrafo durante la aplicación de radiofrecuencia. Se observa la presencia de dos extrasístoles ventriculares, seguidas de ritmo sinusal y cuatro latidos de taquicardia ventricular que degeneran en fibrilación ventricular.

interacción de sus componentes: anillo mitral, valvas, aparato subvalvular y músculos papilares. El anillo mitral es una estructura tridimensional en forma de silla de montar con cambios conformacionales pasivos durante el ciclo cardiaco. La movilidad del anillo está determinada por la contracción y la relajación de los músculos atrial y ventricular adyacentes ${ }^{9}$. Las valvas están articuladas desde el anillo fibroso; sin embargo, existen diferencias significativas en la disposición de la articulación en los segmentos aórtico (anterior) y mural (posterior) ${ }^{10}$. La valva anterior se encuentra articulada a lo largo del área de continuidad fibrosa engrosada por los trígonos de la válvula aórtica. Este anclaje asegura la continuidad mitroaórtica dentro del ventrículo izquierdo, y confiere a esta parte del anillo mitral mayor aseguramiento y sostén. Además, hace que la probabilidad de dilatación de este segmento sea infrecuente. Por el contrario, la porción mural del anillo (que soporta la valva posterior) se encuentra articulada a la región parietal de la unión atrioventricular izquierda. La configuración del anillo posterior es variada incluso en corazones sin alteraciones anatómicas, y va desde un cordón fibroso firme hasta el reemplazo por tejido fibroadiposo en lugar de un anillo verdadero. Tal configuración hace a esta porción de la estructura mucho más susceptible a la degeneración ${ }^{11}$.

El prolapso mitral se define como un desplazamiento superior de la válvula mitral de al menos $2 \mathrm{~mm}$ durante la sístole y una degeneración mixomatosa de las valvas de la mitral, lo que resulta en un engrosamiento de al menos $5 \mathrm{~mm}$ durante la diástasis. Entre las múltiples consecuencias de esta condición se encuentra la probabilidad de muerte súbita debida a arritmias ventriculares sostenidas, con un riesgo estimado anual que oscila entre el 0,2 y el 1,9\% ${ }^{12}$. La DAM es una anormalidad estructural de origen incierto consistente en una separación anormal entre el anillo valvular mitral (sitio donde se inserta la válvula) y la pared posterolateral basal del miocardio ventricular, que es la región en la que debe implantarse normalmente. Es una anormalidad localizada que afecta el miocardio ventricular que se encuentra justo por debajo de la valva posterior de la mitral, típicamente alrededor de los festones denominados P1 y P2. Dicha condición puede o no acompañarse de prolapso mitral en su definición clásica ${ }^{13}$.

La DAM fue descrita hace más de 30 años en reportes de autopsia de aproximadamente 900 corazones. Se demostró que el $92 \%$ de los pacientes con prolapso 
mitral tenían también DAM². En una revisión sistemática de la literatura reciente se determinaron varios hallazgos: primero, en los pacientes con enfermedad valvular mitral mixomatosa y prolapso mitral, la DAM es común y por lo tanto en estos pacientes es muy importante examinar cuidadosamente la porción posterior del anillo en búsqueda de la disyunción; segundo, la DAM está asociada con la presencia de arritmias ventriculares potencialmente fatales, de tal modo que cuando se detecta son necesarios estudios en profundidad para determinar con mayor precisión el potencial maligno; y tercero, existe evidencia creciente de que en los pacientes sobrevivientes de una muerte súbita sin una causa claramente identificada debe buscarse activamente una DAM, pues esta puede ser la causa del evento arrítmico ${ }^{14}$.

La DAM afecta principalmente el punto de articulación de la valva posterior mitral y condiciona una amplia separación entre el atrio y la pared libre del ventrículo izquierdo ${ }^{15}$. Dicha condición puede ser evaluada en estudios multimodales de imagen no invasiva, como la ecocardiografía $2 \mathrm{D}$ en la vista de eje largo paraesternal durante el fin de la sístole. Recientemente se ha incorporado la ecocardiografía 3D con el fin de evaluar la dinámica anular paradójica con expansión y aplanamiento sistólico secundaria al desacoplamiento de la función anular-ventricular ${ }^{16}$. El estado hipercontráctil y el movimiento curvilíneo anormal de los segmentos basales y mediolaterales del ventrículo izquierdo durante la sístole han sido documentados en estudios de Doppler tisular, en los que se observa un aumento de las velocidades sistólicas que representan un mayor estiramiento del aparato subvalvular y de los músculos papilares adyacentes durante la sístole. Se ha postulado que este movimiento enérgico puede favorecer la generación de complejos ventriculares prematuros y taquicardia ventricular polimórfica asociada con muerte súbita ${ }^{17,18}$.

La presencia de arritmias ventriculares es frecuente y proporcional a la distancia y al área circunferencial comprometida. En términos de relevancia clínica, una distancia mayor de $8,5 \mathrm{~mm}$ se ha relacionado con taquicardia ventricular no sostenida (OR 95\%; IC 1,3-78,1) 19 .

El continuo movimiento anormal y el estrés generado por la anormalidad anatómica llevan al desarrollo de fibrosis, la cual representaría una fase posterior en la evolución de la patología ${ }^{20}$. Así mismo, estaría relacionada con la degeneración del tejido conectivo que más adelante da a la valva el aspecto mixomatoso, altera su plano de coaptación y favorece el prolapso. Se ha documentado la presencia de prolapso mitral hasta en el $78 \%$ de los pacientes con $\mathrm{DAM}^{21}$.
En el caso que se presenta, la caracterización tisular en la resonancia magnética cardiaca no documentó trastornos de perfusión miocárdica en reposo. Sin embargo, se encontraron focos de realce tardío con gadolinio de distribución no coronariana y de localización en el segmento basal inferoseptal e inferolateral a nivel medio ventricular (segmentos 7 y 8), en estrecha relación con la inserción de los músculos papilares. Tales cambios también se observaron en la ecocardiografía intracardiaca. Además, durante el estudio electrofisiológico, tales zonas se relacionaron claramente con las áreas de interés por las características de los potenciales eléctricos. La fibrosis de los músculos papilares y de la región inferobasal del ventrículo izquierdo se ha observado en estudios histopatológicos de pacientes con muerte súbita ${ }^{22}$. Así mismo, existe evidencia de que el realce tardío con gadolinio en los músculos papilares y la distancia longitudinal de la DAM en la pared posterolateral son predictores de eventos arrítmicos potencialmente letales ${ }^{19}$.

Se ha descrito el tratamiento farmacológico con betabloqueadores, bloqueadores de los canales del calcio y otros agentes antiarrítmicos, pero con escaso impacto en la supervivencia actual y en la disminución de la progresión de la enfermedad ${ }^{8,23,24}$. Las técnicas de mapeo y ablación son factibles en pacientes sintomáticos y en aquellos que presentan refractariedad al manejo farmacológico. A menudo, los complejos ventriculares prematuros promueven fibrilación ventricular en estos pacientes.

Las técnicas de ablación pueden reducir la sintomatología y las descargas apropiadas de los desfibriladores. No obstante, el porcentaje de recurrencia de las arritmias (incluyendo aquellas potencialmente fatales) no esta aún establecido y la evidencia actual respalda que los focos pueden surgir del territorio donde no se ha realizado la ablación inicial ${ }^{25}$.

Se ha descrito el potencial proarrítmico durante 0 inmediatamente después de la ablación ${ }^{26,27}$. Syed, et al. reportaron un paciente que desarrolló tormenta arrítmica después de la ablación y varios otros que desarrollaron fibrilación ventricular durante el procedimiento. ${ }^{28} \mathrm{En}$ su estudio se comparan los resultados electrofisiológicos de pacientes con anormalidades valvulares mitrales (prolapso y DAM) que han sufrido un paro cardiaco previo frente a los de pacientes que se encuentran libres de eventos ${ }^{29}$. En este estudio, los pacientes con antecedente de paro cardiaco se caracterizaron por la presencia de extrasístoles ventriculares que surgían del tejido de Purkinje enfermo, y se documentaron potenciales tardíos, mediodiastólicos y fraccionados, de modo 
similar a lo descrito en nuestra paciente. La localización constante de los focos de extrasístole ventricular y el tejido anormal con conducción lenta sugieren una asociación entre alteración estructural y anormalidad de las fibras de Purkinje.

El riesgo de muerte súbita en los pacientes con anormalidades estructurales del aparato valvular mitral ha sido reportado desde su descripción inicial en la década de 1980 hasta la fecha1,8,22. Los mecanismos involucrados en la génesis de las arritmias incluyen mecanismos de reentrada y actividad desencadenada, aunque se encuentra descrito en la literatura el mapeo exitoso y la ablación como arritmias de origen focal ${ }^{30}$. Gracias a los avances tecnológicos y la integración de estudios de imagen multimodal se ha podido entender mejor el proceso fisiopatológico que desencadena las arritmias letales, así como la secuencia evolutiva de eventos. Esto favorece una planeación estratégica y un abordaje integral individualizado en cada paciente.

La corrección quirúrgica de la valvulopatía mitral mixomatosa en pacientes con arritmias ventriculares refractarias al tratamiento farmacológico (aun sin insuficiencia mitral grave) sigue siendo un tema controversial. En algunas series de casos, la disminución en la incidencia de arritmias permitió la reducción o la suspensión del tratamiento farmacológico $22,31-33$. Sin embargo, otra serie encontró que la disminución de la carga arrítmica estaba condicionada por el tiempo de la intervención quirúrgica sobre la válvula. Los pacientes que tuvieron una reducción del $10 \%$ en general tendían a ser más jóvenes, lo que sugiere que una intervención temprana podría modificar el sustrato electrofisiológico ${ }^{34}$.

El implante del desfibrilador se considera en pacientes con características malignas (alto riesgo de muerte súbita), quienes experimentan episodios de taquicardia ventricular sostenida o de fibrilación ventricular. Esto, a pesar de los limitados datos existentes hasta el momento para la utilización de estos dispositivos como prevención primaria. Hasta la fecha no se ha definido con claridad el papel de la ablación de los focos de extrasístoles ventriculares ni de la intervención quirúrgica sobre la válvula mitral en relación con el riesgo de muerte súbita. Son necesarios estudios que permitan determinar si alguna de estas intervenciones puede considerarse de manera general en este grupo de pacientes.

\section{Conclusión}

La DAM es una anormalidad estructural de origen incierto consistente en una separación anormal entre el anillo valvular mitral y la pared posterolateral basal del miocardio ventricular, fuertemente asociada con la presencia de arritmias ventriculares que pueden ser fatales. Cuando se detecta son necesarios estudios en profundidad para determinar con mayor precisión el potencial maligno. La ablación de los focos de extrasístoles ventriculares clínicamente dominantes podría mejorar la sintomatología y reducir las descargas apropiadas del cardiodesfibrilador; sin embargo, es necesario un seguimiento a largo plazo, ya que pueden surgir nuevos focos.

\section{Financiamiento}

Ninguno.

\section{Conflicto de intereses}

Los autores declaran que no tienen ningún conflicto de intereses.

\section{Responsabilidades éticas}

Protección de personas y animales. Los autores declaran que para esta investigación no se han realizado experimentos en seres humanos ni en animales.

Confidencialidad de los datos. Los autores declaran que han seguido los protocolos de su centro de trabajo sobre la publicación de datos de pacientes.

Derecho a la privacidad y consentimiento informado. Los autores declaran que en este artículo no aparecen datos de pacientes.

\section{Bibliografía}

1. Bharati S, Granston AS, Liebson PR, Loeb HS, Rosen KM, Lev M. The conduction system in mitral valve prolapse syndrome with sudden death. Am Heart J. 1981;101:667-70.

2. Hutchins GM, Moore GW, Skoog DK. The association of floppy mitral valve with disjunction of the mitral annulus fibrosus. $\mathrm{N}$ Engl $\mathrm{J}$ Med. 1986;314:535-40.

3. Eriksson MJ, Bitkover CY, Omran AS, David TE, Ivanov J, Ali MJ, et al. Mitral annular disjunction in advanced myxomatous mitral valve disease: echocardiographic detection and surgical correction. J Am Soc Echocardiogr. 2005;18:1014-22.

4. Gilbert BW, Schatz RA, VonRamm OT, Behar VS, Kisslo JA. Mitral valve prolapse. Two-dimensional echocardiographic and angiographic correlation. Circulation. 1976;54:716-23.

5. Noseworthy PA, Asirvatham SJ. The knot that binds mitral valve prolapse and sudden cardiac death. Circulation. 2015;132:551-2.

6. Vaidya VR, DeSimone CV, Damle N, Naksuk N, Syed FF, Ackerman MJ, et al. Reduction in malignant ventricular arrhythmia and appropriate shocks following surgical correction of bileaflet mitral valve prolapse. J Interv Card Electrophysiol. 2016;46:137-43.

7. Sriram CS, Syed FF, Ferguson ME, Johnson JN, Enriquez-Sarano M, Cetta $\mathrm{F}$, et al. Malignant bileaflet mitral valve prolapse syndrome in patients with otherwise idiopathic out-of-hospital cardiac arrest. J Am Coll Cardiol. 2013;62:222-30.

8. Muthukumar L, Jahangir A, Jan MF, Perez Moreno AC, Khandheria BK, Tajik AJ. Association between malignant mitral valve prolapse and sudden cardiac death: a review. JAMA Cardiol. 2020;5:1053-61. 
9. Angelini A, Ho SY, Anderson RH, Davies MJ, Becker AE. A histological study of the atrioventricular junction in hearts with normal and prolapsed leaflets of the mitral valve. Br Heart J. 1988;59:712-6.

10. Ho SY. Anatomy of the mitral valve. Heart. 2002;88 (Suppl 4):iv5-10.

11. Sun F, Chen Y, Huang L, Ren W, Yu X, Ni C. Rare congenital mitra valve malformations assessed by real-time three-dimensional echocardiography. Int J Cardiol. 2016;222:1027-30.

12. Nalliah CJ, Mahajan R, Elliott AD, Haqqani H, Lau DH, Vohra JK, et al. Mitral valve prolapse and sudden cardiac death: a systematic review and meta-analysis. Heart. 2019; 105:144-51.

13. Del Forno B, Castiglioni A, Sala A, Geretto A, Giacomini A, Denti P, et al Mitral valve annuloplasty. Multimed Man Cardiothorac Surg. 2017;2017.

14. Bennett S, Thamman R, Griffiths T, Oxley C, Khan JN, Phan T, et al Mitral annular disjunction: a systematic review of the literature. Echocardiography. 2019;36:1549-58.

15. Carmo P, Andrade MJ, Aguiar C, Rodrigues R, Gouveia R, Silva JA Mitral annular disjunction in myxomatous mitral valve disease: a relevant abnormality recognizable by transthoracic echocardiography. Cardiovasc Ultrasound. 2010;8:53

16. Lee AP, Jin CN, Fan Y, Wong RHL, Underwood MJ, Wan S. Functional implication of mitral annular disjunction in mitral valve prolapse: a quantitative dynamic 3D echocardiographic study. JACC Cardiovasc Imaging. 2017;10:1424-33

17. Perazzolo Marra M, Basso C. Mechanical dispersion and arrhythmic mitral valve prolapse: substrate and trigger in electrical instability. Heart. 2019;105:1053-4

18. Fulton BL, Liang JJ, Enriquez A, Garcia FC, Supple GE, Riley MP, et al Imaging characteristics of papillary muscle site of origin of ventricula arrhythmias in patients with mitral valve prolapse. J Cardiovasc Electrophysiol. 2018;29:146-53.

19. Bennett S, Thamman R, Griffiths T, Oxley C, Khan JN, Phan T, et al Mitral annular disjunction: a systematic review of the literature. Echocardiography. 2019;36:1549-58.

20. Chahal NS, Lim TK, Jain P, Chambers JC, Kooner JS, Senior R. Normative reference values for the tissue Doppler imaging parameters of left ventricular function: a population-based study. Eur J Echocardiogr. 2010;11:51-6.

21. Dejgaard LA, Skjølsvik ET, Lie $\varnothing$, Ribe M, Stokke MK, Hegbom F, et al. The mitral annulus disjunction arrhythmic syndrome. J Am Coll Cardiol. 2018;72:1600-9.

22. Basso C, Perazzolo Marra M, Rizzo S, De Lazzari M, Giorgi B, Cipriani A et al. Arrhythmic mitral valve prolapse and sudden cardiac death. Circulation. 2015;132:556-66.
23. Wilde AA, Düren DR, Hauer RN, deBakker JM, Bakker PF, Becker AE et al. Mitral valve prolapse and ventricular arrhythmias: observations in a patient with a 20-year history. J Cardiovasc Electrophysiol. 1997; 8:307-16.

24. Vohra J, Sathe S, Warren R, Tatoulis J, Hunt D. Malignant ventricular arrhythmias in patients with mitral valve prolapse and mild mitral regurgitation. Pacing Clin Electrophysiol. 1993:16(3 Pt 1):387-93.

25. Syed FF, Ackerman MJ, McLeod CJ, Kapa S, Mulpuru SK, Sriram CS, et al. Sites of successful ventricular fibrillation ablation in bileaflet mitral valve prolapse syndrome. Circ Arrhythm Electrophysiol. 2016;9:e004005.

26. Wang Q, Madhavan M, Viqar-Syed M, Asirvatham SJ. Successful ablation of a narrow complex tachycardia arising from a left ventricular false tendon: mapping and optimizing energy delivery. Heart Rhythm. 2014; 11:321-4.

27. Hai JJ, Desimone CV, Vaidya VR, Asirvatham SJ. Endocavitary structures in the outflow tract: anatomy and electrophysiology of the conus papillary muscles. J Cardiovasc Electrophysiol. 2014;25:94-8.

28. Syed FF, Ackerman MJ, McLeod CJ, Kapa S, Mulpuru SK, Sriram CS et al. Sites of successful ventricular Ffibrillation ablation in Bileaflet mitral valve prolapse syndrome. Circ Arrhythm Electrophysiol. 2016 May; 9(5):e004005.

29. Van Herendael H, Zado ES, Haqqani H, Tschabrunn CM, Callans DJ, Frankel DS, et al. Catheter ablation of ventricular fibrillation: importance of left ventricular outflow tract and papillary muscle triggers. Heart Rhythm. 2014:11:566-73.

30. Noda T, Shimizu W, Taguchi A, Aiba T, Satomi K, Suyama K, et al. Malignant entity of idiopathic ventricular fibrillation and polymorphic ventricular tachycardia initiated by premature extrasystoles originating from the right ventricular outflow tract. J Am Coll Cardiol. 2005;46:1288-94.

31. Scheirlynck E, Dejgaard LA, Skjølsvik E, Lie OH, Motoc A, Hopp E, et al. Increased levels of SST2 in patients with mitral annulus disjunction and ventricular arrhythmias. Open Heart. 2019;6:e001016.

32. Abbadi DR, Purbey R, Poornima IG. Mitral valve repair is an effective treatment for ventricular arrhythmias in mitral valve prolapse syndrome. Int J Cardiol. 2014:177:e16-8.

33. Pocock WA, Barlow JB, Marcus RH, Barlow CW. Mitral valvuloplasty for life-threatening ventricular arrhythmias in mitral valve prolapse. Am Heart J. 1991;121(1 Pt 1):199-202.

34. Naksuk N, Syed FF, Krittanawong C, Anderson MJ, Ebrille E, DeSimone CV, et al. The effect of mitral valve surgery on ventricular arrhythmia in patients with bileaflet mitral valve prolapse. Indian Pacing Electrophysiol J. 2016;16:187-91. 